\title{
On distant-isomorphisms of projective lines
}

\author{
Andrea Blunck Hans Havlicek
}

\begin{abstract}
We determine all distant-isomorphisms between projective lines over semilocal rings. In particular, for those semisimple rings that do not have a simple component which is isomorphic to a field, every distant isomorphism arises from a Jordan isomorphism of rings and a projectivity. We show this by virtue of a one-one correspondence linking the projective line over a semisimple ring with a Segre product of Grassmann spaces.

Mathematics Subject Classification (2000). 51C05, 51A10, 51A45, $17 \mathrm{C} 50$.

Key Words. projective line over a ring, distant-isomorphism, Jordan homomorphism, Grassmann space, Segre product.
\end{abstract}

\section{Introduction.}

1.1. The projective line over a (skew) field is often said to have no intrinsic structure. However, if we consider a ring $R$ other than a field, then the projective line $\mathbb{P}(R)$ carries a non-trivial relation "distant". Intuitively, points $p$ and $q$ of $\mathbb{P}(R)$ are distant (in symbols: $p \Delta q$ ) if $p$ and $q$ span the entire projective line, whereas non-distant points are "too close together" in order to share this property. Therefore non-distant points are also called neighbouring. If $R$ is a field, then $p \triangle q$ just means that $p \neq q$, whence in this case the distant relation does not deserve our interest, and $\mathbb{P}(R)$ indeed has no intrinsic structure.

For rings that are not fields, however, the following question is interesting: Which bijections between projective lines over rings $R$ and $R^{\prime}$ map distant points to distant points and non-distant points to non-distant points? Every mapping of this kind will be called a distant-isomorphism. It seems that no attention has been paid to this question so far. One reason could be that we cannot expect an algebraic description of distant-isomorphisms for some classes of rings like fields, direct products of fields, or local rings. Here, loosely speaking, "algebraic description" means that the mapping under consideration is the product of a bijection $\mathbb{P}(R) \rightarrow \mathbb{P}\left(R^{\prime}\right)$ which stems from a Jordan isomorphism $R \rightarrow R^{\prime}$ and a projectivity of $\mathbb{P}\left(R^{\prime}\right)$.

In [6] it was shown that such an algebraic description of distant-isomorphisms is possible if both $R$ and $R^{\prime}$ are rings of $2 \times 2$ matrices over fields. In the present 
note we extend this result to the case when both $R$ and $R^{\prime}$ are direct products of matrix rings over fields, provided that no ring of $1 \times 1$ matrices is present in these products (Theorem 6.7).

In order to reach this goal we bring together several concepts and results. Some basic facts about the projective line $\mathbb{P}(R)$ are presented in Section 2. In Section 3 we recall the definition and some properties of a parallelism (written as $\|$ ) on $\mathbb{P}(R)$; it was introduced in [10], and it reflects the Jacobson radical of $R$ in geometric terms. This relation $\|$ is, by its definition, invariant under distant-isomorphisms. At this point we have to emphasize that many authors, like Herzer in [16], use the symbol \| and the phrase "parallel points" in a different meaning, namely for what we simply call non-distant points. Next, we introduce an adjacency relation (written as $\sim$ ) on $\mathbb{P}(R)$. This notion comes from the geometry of Grassmann spaces. It is well known that the projective line over a ring of matrices with entries from a field is in one-one correspondence with the points of some Grassmann space. In a very general form, this is a result about the projective line over the endomorphism ring of a (possibly infinite-dimensional) vector space; see [4]. Clearly, we could use this one-one correspondence in order to define an adjacency relation on $\mathbb{P}(R)$ as the preimage of the adjacency relation on the associated Grassmann space. However, we use an intrinsic definition of adjacency on $\mathbb{P}(R)$ in terms of the distant relation; the idea for this definition is taken from [11].

In Section 4 we show that one cannot expect an algebraic description of distantisomorphisms for rings with a non-zero Jacobson radical, because for these rings one has a non-trivial parallelism, and every permutation of $\mathbb{P}(R)$ that maps each point to a parallel one is a distant-automorphism. Then, in Section 5, we establish our main result in a first step for matrix rings over fields (Theorem 5.4); this is generalized in Section 6 to semisimple rings, i.e. direct products of finitely many matrix rings over fields (Theorem 6.7). Altogether, the results from Sections 4, 5, and 6 give a complete description of the distant-isomorphisms for semilocal rings, i.e. rings which are semisimple modulo their Jacobson radical (Corollary 6.8).

In Sections 5 and 6 we use Grassmann spaces and their Segre products to represent the projective lines over semisimple rings in terms of partial linear spaces. In this setting, distant-isomorphisms correspond to collineations of these spaces (Propositions 5.2 and 6.6), and the adjacency relations are the key to all this. Then we can use a recent result by Naumowicz and Prażmowski (see [23]) in order to accomplish our work. As a matter of fact, we need their result in a slightly generalized form, which is presented as an Appendix to this article.

Alternatively, our results can also be viewed as a generalization of Chow's theorem [13] on adjacency preserving bijections of Grassmann spaces to Segre products of such spaces. Cf. also [3], [17], and [26].

There is a wealth of literature on harmonic mappings for projective lines over rings. A survey is given by Bartolone and Bartolozzi [2], and Lashkhi [22]; see also [9]. As every harmonic mapping takes distant points to distant points, our results 
may also be viewed as a contribution to this topic. However, this connection will be discussed elsewhere.

1.2. Throughout this paper we shall only consider associative rings with a unit element $1 \neq 0$, which is preserved by (anti-)homomorphisms, inherited by subrings, and acts unitally on modules. The group of invertible elements (units) of a ring $R$ is denoted by $R^{*}$. Moreover, by a field we always mean a not necessarily commutative field. We refer to [20] for those notions and results from ring theory which are used in the text without reference.

\section{Distant-morphisms.}

2.1. Consider a ring $R$ and the free left $R$-module $R^{2}$. The projective line over $R$ is the orbit of the free cyclic submodule $R(1,0)$ under the natural right action of the group $\mathrm{GL}_{2}(R)$ of invertible $2 \times 2$ matrices with entries in $R$. In other words, $\mathbb{P}(R)$ is the set of all $p \leq R^{2}$ such that $p=R(a, b)$, where $(a, b)$ is the first row of an invertible matrix. See [16, p. 785]. If also $(c, d)$ is the first row of an invertible matrix, then $R(a, b)=R(c, d)$ if, and only if, there is a unit $u \in R^{*}$ with $(c, d)=u(a, b)$ (see [5, Prop. 2.1]).

Let $((a, b),(c, d))$ be a basis of $R^{2}$ or, said differently, let $\left(\begin{array}{ll}a & b \\ c & d\end{array}\right) \in \mathrm{GL}_{2}(R)$. Then the points $p=R(a, b)$ and $q=R(c, d)$ are called distant, and we write $p \triangle q$. Obviously, $\triangle$ is an anti-reflexive and symmetric relation on $\mathbb{P}(R)$.

In the special case that $R$ is a field, $\mathbb{P}(R)$ is the usual projective line over $R$, and $\Delta$ is the relation $\neq$.

The projective line $\mathbb{P}(R)$ together with the relation $\Delta$ can also be seen as a graph (the distant graph) with vertex set $\mathbb{P}(R)$ and two vertices joined by an edge if, and only if, they are distant. See [7] for graph theoretic properties of $(\mathbb{P}(R), \triangle)$.

For each point $p \in \mathbb{P}(R)$ we let $\triangle(p)$ be the neighbourhood of $p$ in the distant graph, i.e. the set of all points distant to $p$.

2.2. We are interested in mappings preserving the distant relation. Let $R$ and $R^{\prime}$ be rings, and let $\varphi: \mathbb{P}(R) \rightarrow \mathbb{P}\left(R^{\prime}\right)$ be a mapping with

$$
\forall p, q \in \mathbb{P}(R): p \triangle q \Rightarrow p^{\varphi} \triangle q^{\varphi},
$$

where, by abuse of notation, the distant relation on $\mathbb{P}\left(R^{\prime}\right)$ is denoted by $\triangle$ rather than $\triangle^{\prime}$. Then we call $\varphi$ a distant-morphism (or $\triangle$-morphism). So the $\Delta$-morphisms are exactly the homomorphisms of our distant graphs. If $\varphi$ is a bijective $\triangle$-morphism and also $\varphi^{-1}$ is a $\Delta$-morphism then, as usual, we call $\varphi$ a $\triangle$-isomorphism.

2.3 Remark. It is worth noting that a bijective $\triangle$-morphism need not be a $\triangle$-isomorphism. Indeed, let $\mathbb{Z}$ be the ring of integers and let $\mathbb{Q}$ be the field of rational 
numbers. A pair $(a, b) \in \mathbb{Z}^{2}$ is the first row of an invertible matrix if, and only if, it is unimodular, i.e., if there are integers $x$ and $y$ such that $a x+b y=1[16$, Prop. 1.4.1]. On the other hand, each point of $\mathbb{P}(\mathbb{Q})$ can be written in the form $\mathbb{Q}(a, b)$ with an unimodular pair $(a, b) \in \mathbb{Z}^{2}$. In either case two unimodular pairs of integers yield the same point precisely when they are proportional by a factor \pm 1 . Therefore the mapping $\varphi: \mathbb{P}(\mathbb{Z}) \rightarrow \mathbb{P}(\mathbb{Q}): \mathbb{Z}(a, b) \mapsto \mathbb{Q}(a, b)$ is well defined and bijective. Condition (1) is satisfied, as here $p^{\varphi} \triangle q^{\varphi}$ just means $p^{\varphi} \neq q^{\varphi}$. However, by $\mathbb{Z}(1,0) \not \mathbb{Z}(1,2)$ and $\mathbb{Q}(1,0) \neq \mathbb{Q}(1,2)$, the bijection $\varphi$ is not a $\triangle$-isomorphism.

Cf. Remark 1 in [2, p. 359] for another example of such a bijective $\triangle$-morphism.

2.4. There are several classes of examples of $\triangle$-morphisms:

(a) By definition, the group $\mathrm{GL}_{2}(R)$ acts (transitively) on $\mathbb{P}(R)$ and leaves $\triangle$ invariant. So, for each $\gamma \in \mathrm{GL}_{2}(R)$, the induced projectivity $\widetilde{\gamma}: \mathbb{P}(R) \rightarrow \mathbb{P}(R)$ : $R(a, b) \mapsto R\left((a, b)^{\gamma}\right)$ is a $\triangle$-automorphism.

(b) Each ring homomorphism $\alpha: R \rightarrow R^{\prime}$ gives rise to a mapping

$$
\widetilde{\alpha}: \mathbb{P}(R) \rightarrow \mathbb{P}\left(R^{\prime}\right): R(a, b) \mapsto R^{\prime}\left(a^{\alpha}, b^{\alpha}\right) .
$$

One can easily check that $\widetilde{\alpha}$ maps distant points to distant points and hence is a $\triangle$-morphism. In particular, if $\alpha$ is an isomorphism of rings, then $\widetilde{\alpha}$ is a $\Delta$-isomorphism.

(c) Let $\alpha: R \rightarrow R^{\prime}$ be an anti-homomorphism of rings. In general, $\widetilde{\alpha}$ as in (2) is not well defined any more. Instead, we may proceed as follows. For each point $p$ there is a regular matrix $M$ with first row $(a, b)$, say, and $p=R(a, b)$. Let $(v, w)^{\mathrm{T}}$, where $\mathrm{T}$ denotes transposition of matrices, be the second column of $M^{-1}$. Then we define

$$
\widetilde{\alpha}: \mathbb{P}(R) \rightarrow \mathbb{P}\left(R^{\prime}\right): p \mapsto R^{\prime}\left(-w^{\alpha}, v^{\alpha}\right) .
$$

This is a well defined $\triangle$-morphism, as follows from the proof of [8, Thm. 5.2] and [8, Rem. 5.4], where all this is studied in the context of chain geometries and dual pairs of $R$-modules. (For finite-dimensional algebras this result is due to Herzer [15, Prop. 3.3].)

Suppose that $\alpha$ is also a homomorphism, whence the $\operatorname{ring} R^{\alpha}$ is commutative. So we may apply the cofactor method to invert matrices with coefficients in $R^{\alpha}$. By calculating the second column of $\left(M^{-1}\right)^{\alpha}=\left(M^{\alpha}\right)^{-1}$ in two ways, we get $\left(v^{\alpha}, w^{\alpha}\right)^{\mathrm{T}}=\left(\operatorname{det} M^{\alpha}\right)^{-1}\left(-b^{\alpha}, a^{\alpha}\right)^{\mathrm{T}}$. Thus, the definitions in (2) and (3) coincide, and it is unambiguous to use the same symbol $\widetilde{\alpha}$ in either case.

(d) Now consider a Jordan homomorphism $R \rightarrow R^{\prime}$, i.e. an additive mapping $\alpha$ satisfying $1^{\alpha}=1 \in R^{\prime}$ and $(a b a)^{\alpha}=a^{\alpha} b^{\alpha} a^{\alpha}$ for all $a, b \in R$. Of course, each homomorphism or anti-homomorphism is also a Jordan homomorphism. See Corollary 6.9 and $[9,3.8]$ for examples of Jordan homomorphisms that are neither homomorphisms nor anti-homomorphisms.

In $[9$, Thm. 4.4 (b)] it is shown that each Jordan homomorphism gives rise to at least one $\triangle$-morphism. However, the definition itself is rather involved, and we do not need it here. Instead, we present a special case, due to Bartolone. 
Let $R$ be a ring of stable rank 2 (for the definition see [25, p. 1039]). Then

$$
\mathbb{P}(R)=\{R(a b-1, a) \mid a, b \in R\} .
$$

If, moreover, $R^{\prime}$ is any ring and $\alpha: R \rightarrow R^{\prime}$ is a Jordan homomorphism, then

$$
\widetilde{\alpha}: R(a b-1, a) \mapsto R^{\prime}\left(a^{\alpha} b^{\alpha}-1, a^{\alpha}\right)
$$

is a $\triangle$-morphism $\mathbb{P}(R) \rightarrow \mathbb{P}\left(R^{\prime}\right)$. See $[1$, Thm. (2.4)]. If $\alpha$ is a homomorphism or an anti-homomorphism, then $\widetilde{\alpha}$ coincides with the mapping of (2) or (3), respectively. In case of a homomorphism this is obvious, otherwise we refer to [8, Rem. 5.4].

\section{Adjacency.}

3.1. The distant relation on $\mathbb{P}(R)$ yields two further binary relations. First we recall the following definition from [10]: Consider $p, q \in \mathbb{P}(R)$. We say that $p$ and $q$ are parallel if

$$
\triangle(p) \subseteq \triangle(q) .
$$

In this case we write $p \| q$. In [10, Cor. 2.3] it is shown that $\|$ (called radical parallelism there) is an equivalence relation. So

$$
p \| q \Leftrightarrow \triangle(p)=\triangle(q) .
$$

For $p, q \in \mathbb{P}(R)$ we always have

$$
p \| q \Rightarrow p \not \triangleleft q,
$$

because otherwise $q \in \Delta(p) \subseteq \triangle(q)$ in contradiction to $q \not \not q$.

The Jacobson radical $\operatorname{rad} R$ of the ring $R$ is the intersection of all maximal left (or all maximal right) ideals of $R$. It is a two-sided ideal of $R$, whence one can consider the canonical epimorphism

$$
\pi: R \rightarrow \bar{R}:=R / \operatorname{rad} R: a \mapsto \bar{a}:=a+\operatorname{rad} R .
$$

According to (2), we obtain the associated $\triangle$-morphism

$$
\widetilde{\pi}: \mathbb{P}(R) \rightarrow \mathbb{P}(\bar{R}): p \mapsto \bar{p}:=p^{\tilde{\pi}}
$$

which is surjective (see [5, Prop. 3.5]). By [10, Thm. 2.2], for $p, q \in \mathbb{P}(R)$ we have

$$
p \| q \Leftrightarrow \bar{p}=\bar{q} .
$$


3.2. We call the points $p, q \in \mathbb{P}(R)$ adjacent, and write $p \sim q$, if

$$
\exists r \in \mathbb{P}(R): r \not \mid p, q \text { and } \triangle(r) \subseteq \triangle(p) \cup \triangle(q) .
$$

In this situation we also say that $p$ is adjacent to $q$ via $r$.

Obviously, the relation $\sim$ is symmetric and anti-reflexive; we even have $p \sim q \Rightarrow$ $p \nVdash q$, since otherwise, if $p \sim q$ via $r$, we had $\Delta(r) \subseteq \Delta(p) \cup \Delta(q)=\Delta(p)$ and hence $r \| p$, which is not allowed by (12). A description of the relation $\sim$ for arbitrary rings is beyond the scope of this article. However, in 3.7 and Proposition 3.8 we shall show where this definition has its origin.

The next lemma says how $\Delta$ and $\sim$ behave on parallel classes.

3.3 Lemma. Let $p_{1}, q_{1}, r_{1}, p_{2}, q_{2}, r_{2} \in \mathbb{P}(R)$ with $p_{1}\left\|p_{2}, q_{1}\right\| q_{2}$, and $r_{1} \| r_{2}$. Then

(a) $p_{1} \triangle q_{1} \Leftrightarrow p_{2} \triangle q_{2}$,

(b) $p_{1} \sim q_{1}$ via $r_{1} \Leftrightarrow p_{2} \sim q_{2}$ via $r_{2}$.

Proof. (a): Since $p_{1} \| p_{2}$, we get from $p_{1} \triangle q_{1}$ that $q_{1} \triangle p_{2}$. Then, since $q_{1} \| q_{2}$, we get from $q_{1} \triangle p_{2}$ that $p_{2} \triangle q_{2}$.

(b): Let $p_{1} \sim q_{1}$ via $r_{1}$, i.e., $r_{1} \nVdash p_{1}, q_{1}$ and $\Delta\left(r_{1}\right) \subseteq \triangle\left(p_{1}\right) \cup \triangle\left(q_{1}\right)$. Transitivity of $\|$ yields $r_{2} \nVdash p_{2}, q_{2}$. Moreover, $\Delta\left(r_{2}\right)=\Delta\left(r_{1}\right) \subseteq \Delta\left(p_{1}\right) \cup \triangle\left(q_{1}\right)=\Delta\left(p_{2}\right) \cup \triangle\left(q_{2}\right)$.

This lemma means that the relations $\Delta$ and $\sim$ are well defined on the set of parallel classes of $\mathbb{P}(R)$. By (11), this can also be formulated in terms of the surjective mapping $\widetilde{\pi}$ onto the projective line over $\bar{R}=R / \operatorname{rad} R$ :

3.4 Corollary. Let $\tilde{\pi}: \mathbb{P}(R) \rightarrow \mathbb{P}(\bar{R}): p \mapsto \bar{p}$ be as in (10). Then for all $p, q, r \in$ $\mathbb{P}(R)$ we have

(a) $p \triangle q \Leftrightarrow \bar{p} \triangle \bar{q}$,

(b) $p \sim q$ via $r \Leftrightarrow \bar{p} \sim \bar{q}$ via $\bar{r}$.

Assertion (a) can also be shown algebraically, see [5, Props. 3.1, 3.2].

3.5 Remark. As an example, we study the three relations $\Delta, \|$, and $\sim$ for local rings, i.e. rings $R$ where the set $R \backslash R^{*}$ of non-units is an ideal. Then $R \backslash R^{*}$ is the unique maximal ideal of $R$ and coincides with the Jacobson radical.

For an arbitrary ring $R$ the following characterizations were established in [16, Prop. 2.4.1] and [10, Thm. 2.5]: $R$ is local if, and only if, $\not$ is an equivalence relation on $\mathbb{P}(R)$, which in turn is equivalent to

$$
\forall p, q \in \mathbb{P}(R): p \not \not q \Leftrightarrow p \| q .
$$


We claim that for a local ring $R$ we have

$$
\forall p, q \in \mathbb{P}(R): p \triangle q \Leftrightarrow p \sim q .
$$

In fact, we infer from Corollary 3.4 (a) that $p \Delta q \Leftrightarrow \bar{p} \Delta \bar{q}$. Since $\bar{R}$ is a field, we can rewrite the last condition as $\bar{p} \neq \bar{q}$. Taking into account that $\mathbb{P}(\bar{R})$ contains at least three different points, we see that $\bar{p} \neq \bar{q} \Leftrightarrow \bar{p} \sim \bar{q}$ (via any point $\bar{r} \neq \bar{p}, \bar{q}$ ). By Corollary 3.4 (b), this is equivalent to $p \sim q$.

3.6. Let us recall the concept of a partial linear space (or semilinear space or partial line space): This is a point-line geometry $\mathfrak{M}=(\mathcal{P}, \mathcal{L})$, where any two distinct points are joined by at most one line, and every line contains at least two distinct points. Note that we do not make any richness conditions on $\mathcal{P}$ or $\mathcal{L}$, as some authors do. If points are on a common line then they are said to be collinear. A strong subspace (or linear subspace) of $\mathfrak{M}$ is a set $\mathcal{S} \subseteq \mathcal{P}$ of mutually collinear points such that $\mathcal{S}$ is closed under lines. Following [23], a partial linear space $\mathfrak{M}$ is called strongly connected if for every point $p \in \mathcal{P}$ and every strong subspace $\mathcal{S} \subseteq \mathcal{P}$, with $\# \mathcal{S}>1$, there is a finite sequence $\mathcal{S}_{0}, \mathcal{S}_{1}, \ldots, \mathcal{S}_{m}$ of strong subspaces such that $\mathcal{S}=\mathcal{S}_{0}, p \in \mathcal{S}_{m}$ and $\#\left(\mathcal{S}_{i-1} \cap \mathcal{S}_{i}\right) \geq 2$ for all $i \in\{1,2, \ldots, m\}$.

3.7. Let $\boldsymbol{U}$ be a left vector space over a field $K, \operatorname{dim} \boldsymbol{U} \geq 1$, and let $R=\operatorname{End}_{K}(\boldsymbol{U})$ be its endomorphism ring. We restrict ourselves to the case when $n:=\operatorname{dim} \boldsymbol{U}$ is finite, even though most of the subsequent results can be formulated in such a way that they remain valid also in case of infinite dimension.

The following is taken from [4, Thm. 2.4], whereas in [5] the viewpoint of projective geometry was adopted: Let $\mathcal{G}$ be the set of all $n$-dimensional subspaces of the vector space $\boldsymbol{U} \times \boldsymbol{U}$. Then

$$
\Psi: \mathbb{P}(R) \rightarrow \mathcal{G}: R(a, b) \mapsto \boldsymbol{U}^{(a, b)}:=\left\{\left(\boldsymbol{u}^{a}, \boldsymbol{u}^{b}\right) \mid \boldsymbol{u} \in \boldsymbol{U}\right\}
$$

is a well defined bijection mapping distant points of $\mathbb{P}(R)$ to complementary subspaces in $\mathcal{G}$ and non-distant points to non-complementary subspaces. Moreover, the groups $\mathrm{GL}_{2}(R)$ and $\mathrm{Aut}_{K}(\boldsymbol{U} \times \boldsymbol{U})$ are isomorphic, and their actions on $\mathbb{P}(R)$ and on $\mathcal{G}$, respectively, are equivalent via $\Psi$.

On $\mathcal{G}$ there is a binary relation

$$
\boldsymbol{P} \sim \boldsymbol{Q}: \Leftrightarrow \operatorname{dim}(\boldsymbol{P} \cap \boldsymbol{Q})=n-1 \Leftrightarrow \operatorname{dim}(\boldsymbol{P}+\boldsymbol{Q})=n+1,
$$

where $\sim$ is to be read as adjacent. It has been studied by many authors. See, for example, [13], [17], and [26]. The graph with vertex set $\mathcal{G}$ and two vertices $\boldsymbol{P}, \boldsymbol{Q}$ joined by an edge if, and only if, $\boldsymbol{P} \sim \boldsymbol{Q}$ is called the Grassmann graph on $\mathcal{G}$. Compare, among others, [12], [14], and [24].

The set $\mathcal{G}$ is the point set of the Grassmann space $\mathfrak{M}=(\mathcal{G}, \mathcal{L})$, with line set $\mathcal{L}$ consisting of all pencils

$$
\mathcal{G}[M, N]:=\{\boldsymbol{X} \in \mathcal{G} \mid \boldsymbol{M} \subset \boldsymbol{X} \subset \boldsymbol{N}\},
$$


where $\operatorname{dim} \boldsymbol{M}=n-1, \operatorname{dim} \boldsymbol{N}=n+1$, and $\boldsymbol{M} \subset \boldsymbol{N}$. The Grassmann space $\mathfrak{M}$ has at least one line and at least three points on every line. It is a strongly connected partial linear space; see [23, Prop. 1.13], where this is shown for a wider class of Grassmann spaces. (In [23] the term spaces of pencils is used instead. If $\boldsymbol{U}$ has infinite dimension then there are points which cannot be joined by a (finite) polygonal path, i.e., the partial linear space $\mathfrak{M}$ is not even connected.)

The following result is crucial for the entire paper:

3.8 Proposition. Let $R=\operatorname{End}_{K}(\boldsymbol{U})$ be the endomorphism ring of an $n$ dimensional left vector space $\boldsymbol{U}$ over a field $K, 1 \leq n<\infty$, let $\mathfrak{M}$ be the associated Grassmann space, and let $\Psi: \mathbb{P}(R) \rightarrow \mathcal{G}$ be given as in (15). Then the following statements hold for all $p, q$, and $r \in \mathbb{P}(R)$ :

(a) $p \sim q \Leftrightarrow p^{\Psi} \sim q^{\Psi} \Leftrightarrow p^{\Psi}$ and $q^{\Psi}$ are distinct collinear points of $\mathfrak{M}$.

(b) $p \sim q$ via $r \Leftrightarrow p^{\Psi}, q^{\Psi}$, and $r^{\Psi}$ are distinct collinear points of $\mathfrak{M}$.

Proof. Note that in the present situation the condition $r \not p p, q$ in (12) reduces to $r \neq$ $p, q$, because $R=\operatorname{End}_{K}(\boldsymbol{U})$ is simple, whence $\operatorname{rad} R=\{0\}$, and parallelity means equality by (11). Now the first equivalence in (a) is an immediate consequence of [11, Thm. 3.2]. Furthermore, $p^{\Psi} \sim q^{\Psi}$ just means that $p^{\Psi} \neq q^{\Psi}$ both belong to a line of $\mathfrak{M}$, namely $\mathcal{G}\left[p^{\Psi} \cap q^{\Psi}, p^{\Psi}+q^{\Psi}\right]$.

For the assertion in (b) we refer to the proof of [11, Thm. 3.2].

\section{Distant-isomorphisms.}

4.1. After having seen in 2.4 how to construct $\triangle$-morphisms from given algebraic mappings, we now aim at a description of all $\triangle$-isomorphisms. Clearly, every $\triangle$-isomorphism is also a $\|$-isomorphism and a $\sim$-isomorphism, where $\|$-morphisms and $\sim$-morphisms are defined like $\triangle$-morphisms.

4.2 Remark. Note that an injective $\Delta$-morphism $\varphi$ need not be a $\|$-morphism. Take, e.g., the ring $R=K(\varepsilon)$ (with $\varepsilon^{2}=0$ ) of dual numbers over a field $K$. The right regular representation of $R$ is the monomorphism $\alpha: R \rightarrow \operatorname{End}_{K}(R): a \mapsto$ $(x \mapsto x a)$. The associated mapping $\widetilde{\alpha}$ is injective, whence it maps the parallel points $R(1,0)$ and $R(1, \varepsilon)$ to distinct points, which are non-parallel as $\operatorname{End}_{K}(R)$ has zero radical.

4.3. In a first step we describe $\|$-isomorphisms. Let $R$ and $R^{\prime}$ be rings. By formula (14) in $[10$, p. 118] we obtain $\# \operatorname{rad} R=\# \mathcal{C}$ for every parallel class $\mathcal{C} \subset \mathbb{P}(R)$. Recall that according to (11) the set of parallel classes of $\mathbb{P}(R)$ can be identified via (10) with $\mathbb{P}(\bar{R})$, where $\bar{R}=R / \operatorname{rad} R$. Hence there exists a $\|$-isomorphism $\mathbb{P}(R) \rightarrow \mathbb{P}\left(R^{\prime}\right)$ if, and only if,

$$
\# \operatorname{rad} R=\# \operatorname{rad} R^{\prime} \text { and } \# \mathbb{P}(\bar{R})=\# \mathbb{P}\left(\overline{R^{\prime}}\right) .
$$


Provided that these conditions are satisfied, the description of all $\|$-isomorphisms $\mathbb{P}(R) \rightarrow \mathbb{P}\left(R^{\prime}\right)$ is a trivial task: If we fix one $\|$-isomorphism $\varphi_{0}: \mathbb{P}(R) \rightarrow \mathbb{P}\left(R^{\prime}\right)$, then every $\|$-isomorphism $\varphi: \mathbb{P}(R) \rightarrow \mathbb{P}\left(R^{\prime}\right)$ has the form $\alpha \varphi_{0}$, where $\alpha$ belongs to the group of $\|$-automorphisms of $\mathbb{P}(R)$. This group in turn is isomorphic to the wreath product of the symmetric group of an arbitrarily chosen parallel class $\mathcal{C}_{0} \subset \mathbb{P}(R)$ and the symmetric group of $\mathbb{P}(\bar{R})$.

For each $\|$-isomorphism $\varphi: \mathbb{P}(R) \rightarrow \mathbb{P}\left(R^{\prime}\right)$ the mapping

$$
\bar{\varphi}: \mathbb{P}(\bar{R}) \rightarrow \mathbb{P}\left(\overline{R^{\prime}}\right): \bar{p} \mapsto \overline{p^{\varphi}}
$$

is a well defined bijection. Observe that every bijection $\mathbb{P}(\bar{R}) \rightarrow \mathbb{P}\left(\overline{R^{\prime}}\right)$ arises in this way. The following is an immediate consequence of Corollary 3.4 (a):

4.4 Proposition. Let $R$ and $R^{\prime}$ be rings. A $\|$-isomorphism $\varphi: \mathbb{P}(R) \rightarrow \mathbb{P}\left(R^{\prime}\right)$ is $a \triangle$-isomorphism if, and only if, the corresponding mapping $\bar{\varphi}$, defined in (19), is $a \triangle$-isomorphism.

4.5. Proposition 4.4 implies that it suffices to study the $\triangle$-isomorphisms $\mathbb{P}(\bar{R}) \rightarrow$ $\mathbb{P}\left(\overline{R^{\prime}}\right)$. However, given a $\triangle$-isomorphism $\varphi: \mathbb{P}(R) \rightarrow \mathbb{P}\left(R^{\prime}\right)$, the mapping $\bar{\varphi}$ only will tell us how $\varphi$ acts on the set of all parallel classes. Unless the radical of $R$ is zero, $\bar{\varphi}$ contains no information at all about the action of $\varphi$ on any parallel class. Every bijection $\beta: \mathbb{P}(R) \rightarrow \mathbb{P}(R)$ which fixes all parallel classes (as sets) is a $\triangle$-automorphism, with $\bar{\beta}$ the identity on $\mathbb{P}(\bar{R})$. Therefore, in general, we cannot expect to describe $\varphi$ "algebraically". However, as above, we have that the group of $\triangle$-automorphisms of $\mathbb{P}(R)$ is isomorphic to the wreath product of the symmetric group of a parallel class $\mathcal{C}_{0}$ and the group of $\triangle$-automorphisms of $\mathbb{P}(\bar{R})$.

We refer to [2, pp. 359-360] for an example of a $\triangle$-automorphism which cannot be described algebraically even though the Jacobson radical is zero: It is based upon the projective line over a polynomial ring $K[X]$, where $K$ is a commutative field with characteristic $\neq 2$. Note that such a ring does not admit proper Jordan endomorphisms [18, pp. 2-3].

4.6. A ring $R$ is called semilocal if $R / \operatorname{rad} R$ is artinian. Since $\operatorname{rad}(R / \operatorname{rad} R)=\{0\}$, this means that $\mathrm{R} / \mathrm{rad} \mathrm{R}$ is semisimple, i.e. artinian with zero radical. By the Wedderburn-Artin theorem, a ring is semisimple if, and only if, it is isomorphic to a direct product of finitely many matrix rings over fields. Note that according to [25, Sec. 2] each semilocal ring has stable rank 2, and observe that in [16] and [25] the terminology is different, as semilocal rings are called semiprimary there.

The results of Sections 5 and 6 together with Proposition 4.4 will give a complete description of the $\triangle$-isomorphisms for projective lines over semilocal rings. See Corollary 6.8. We shall work with endomorphism rings of finite-dimensional vector spaces rather than matrix rings. 


\section{Endomorphism rings.}

5.1. We now study $\triangle$-isomorphisms between the projective lines over endomorphism rings $R=\operatorname{End}_{K}(\boldsymbol{U})$ and $R^{\prime}=\operatorname{End}_{K^{\prime}}\left(\boldsymbol{U}^{\prime}\right)$ of vector spaces $\boldsymbol{U}$ and $\boldsymbol{U}^{\prime}$. We assume throughout this section that

$$
1 \leq \operatorname{dim} \boldsymbol{U}<\infty \text { and } 1 \leq \operatorname{dim} \boldsymbol{U}^{\prime}<\infty .
$$

The sets $\mathcal{G}$ and $\mathcal{G}^{\prime}$ are the point sets of the corresponding Grassmann spaces $\mathfrak{M}$ and $\mathfrak{M}^{\prime}$, respectively. By (15), we have bijections $\Psi: \mathbb{P}(R) \rightarrow \mathcal{G}$ and $\Psi^{\prime}: \mathbb{P}\left(R^{\prime}\right) \rightarrow \mathcal{G}^{\prime}$.

5.2 Proposition. Let $R=\operatorname{End}_{K}(\boldsymbol{U})$ and $R^{\prime}=\operatorname{End}_{K^{\prime}}\left(\boldsymbol{U}^{\prime}\right)$, and let $\varphi: \mathbb{P}(R) \rightarrow$ $\mathbb{P}\left(R^{\prime}\right)$ be a mapping. Then the following statements are equivalent:

(a) $\varphi$ is a $\triangle$-isomorphism.

(b) $\varphi$ is a $\sim$-isomorphism.

(c) $\Psi^{-1} \varphi \Psi^{\prime}: \mathcal{G} \rightarrow \mathcal{G}^{\prime}$ is a collineation $\mathfrak{M} \rightarrow \mathfrak{M}^{\prime}$.

We omit the proof, because this proposition is just that particular case of Proposition 6.6 where $m=m^{\prime}=1$.

5.3. In the following theorem we use the terminology of 2.4. Moreover, we introduce the following notations and conventions: The dual space of $\boldsymbol{U}$, which is a right vector space over $K$, is denoted by $\widehat{\boldsymbol{U}}$. We use the symbol $\langle\cdot, \cdot\rangle$ for the canonical pairing $\boldsymbol{U} \times \widehat{\boldsymbol{U}} \rightarrow K$. For each $a \in \operatorname{End}_{K}(\boldsymbol{U})$ the transpose mapping is written as $a^{\mathrm{T}}$; it is an element of $\operatorname{End}_{K}(\widehat{\boldsymbol{U}})$. The elements of $\widehat{\boldsymbol{U}} \times \widehat{\boldsymbol{U}}$ are considered as columns. Each such column $(\widehat{\boldsymbol{v}}, \widehat{\boldsymbol{w}})^{\mathrm{T}}$ acts as a linear form on $\boldsymbol{U} \times \boldsymbol{U}$ via the formal matrix product

$$
(\boldsymbol{v}, \boldsymbol{w}) \mapsto(\boldsymbol{v}, \boldsymbol{w})\left(\begin{array}{c}
\widehat{\boldsymbol{v}} \\
\widehat{\boldsymbol{w}}
\end{array}\right):=\langle\boldsymbol{v}, \widehat{\boldsymbol{v}}\rangle+\langle\boldsymbol{w}, \widehat{\boldsymbol{w}}\rangle .
$$

This allows us to identify the dual of $\boldsymbol{U} \times \boldsymbol{U}$ with $\widehat{\boldsymbol{U}} \times \widehat{\boldsymbol{U}}$.

5.4 Theorem. Let $R=\operatorname{End}_{K}(\boldsymbol{U})$ and $R^{\prime}=\operatorname{End}_{K^{\prime}}\left(\boldsymbol{U}^{\prime}\right)$. A bijection $\varphi: \mathbb{P}(R) \rightarrow$ $\mathbb{P}\left(R^{\prime}\right)$ is a $\triangle$-isomorphism if, and only if, one of the following conditions is satisfied:

(a) $\operatorname{dim} \boldsymbol{U}=\operatorname{dim} \boldsymbol{U}^{\prime}=1$.

(b) $\operatorname{dim} \boldsymbol{U}>1$ and $\varphi=\widetilde{\alpha} \widetilde{\gamma}$, where $\alpha: R \rightarrow R^{\prime}$ is either an isomorphism or an anti-isomorphism, and $\gamma \in \mathrm{GL}_{2}\left(R^{\prime}\right)$.

Moreover, for $\operatorname{dim} \boldsymbol{U}>1$, the mapping $\alpha$ can be written either as $x \mapsto h^{-1} x h$ with a semilinear bijection $h: \boldsymbol{U} \rightarrow \boldsymbol{U}^{\prime}$, or as $x \mapsto h^{-1} x^{\mathrm{T}} h$ with a semilinear bijection $h: \widehat{\boldsymbol{U}} \rightarrow \boldsymbol{U}^{\prime}$, respectively. 
Proof. Suppose that (a) holds. Then $R \cong K$ and $R^{\prime} \cong K^{\prime}$ so that $\varphi$ is a $\triangle$-isomorphism. If $\varphi$ is given as in (b) then it is a $\triangle$-isomorphism according to 2.4 .

Conversely, let $\varphi$ be a $\triangle$-isomorphism. We read off from [11, Thm. 4.4] that $\operatorname{dim} \boldsymbol{U}=\operatorname{dim} \boldsymbol{U}^{\prime}$, whence we can restrict ourselves to the case $\operatorname{dim} \boldsymbol{U}>1$.

By [11, Thm. 4.4 (c)], applied to $\Psi^{-1} \varphi \Psi^{\prime}$, there are two mutually exclusive possibilities:

(i) There is a semilinear bijection $f: \boldsymbol{U} \times \boldsymbol{U} \rightarrow \boldsymbol{U}^{\prime} \times \boldsymbol{U}^{\prime}$ such that for all $\boldsymbol{X} \in \mathcal{G}$ the image of $\boldsymbol{X}$ under $\Psi^{-1} \varphi \Psi^{\prime}$ equals $\boldsymbol{X}^{f}$.

Choose any semilinear bijection $h: \boldsymbol{U} \rightarrow \boldsymbol{U}^{\prime}$ with the same accompanying isomorphism $K \rightarrow K^{\prime}$ as $f$. Then the mapping $f$ can be written formally as

$$
\left(\boldsymbol{u}_{1}, \boldsymbol{u}_{2}\right) \stackrel{f}{\longmapsto}\left(\boldsymbol{u}_{1}^{h}, \boldsymbol{u}_{2}^{h}\right)\left(\begin{array}{ll}
g_{11} & g_{12} \\
g_{21} & g_{22}
\end{array}\right),
$$

with a matrix $\gamma:=\left(g_{i j}\right) \in \mathrm{GL}_{2}\left(R^{\prime}\right)$; cf. [21, pp. 642-643]. This $\gamma$ is at the same time a linear bijection of $\boldsymbol{U}^{\prime} \times \boldsymbol{U}^{\prime}$; cf. 3.7. Clearly, $\alpha: R \rightarrow R^{\prime}: x \mapsto h^{-1} x h$ is an isomorphism of rings. A straightforward calculation yields

$$
\left(\boldsymbol{U}^{(a, b)}\right)^{f}=\left(\boldsymbol{U}^{\prime\left(a^{\alpha}, b^{\alpha}\right)}\right)^{\gamma} \text { for all }(a, b) \in R^{2} \text { with } R(a, b) \in \mathbb{P}(R) .
$$

Consequently, $R(a, b)^{\varphi}=R(a, b)^{\tilde{\alpha} \widetilde{\gamma}}$, as required.

(ii) There is a semilinear bijection $f: \widehat{\boldsymbol{U}} \times \widehat{\boldsymbol{U}} \rightarrow \boldsymbol{U}^{\prime} \times \boldsymbol{U}^{\prime}$ such that for all $\boldsymbol{X} \in \mathcal{G}$ the image of $\boldsymbol{X}$ under $\Psi^{-1} \varphi \Psi^{\prime}$ equals $\left(\boldsymbol{X}^{\perp}\right)^{f}$, where $\boldsymbol{X}^{\perp}$ denotes the annihilator of $\boldsymbol{X}$.

Let $R(a, b) \in \mathbb{P}(R)$ be a point. There are $c, d \in R$ such that $M:=\left(\begin{array}{ll}a & b \\ c & d\end{array}\right)$ is invertible. Let $(v, w)^{\mathrm{T}}$ be the second column of $M^{-1}$. We claim that

$$
\boldsymbol{U}^{(a, b) \perp}=\left\{\left(\begin{array}{c}
\widehat{\boldsymbol{u}}^{v^{\mathrm{T}}} \\
\widehat{\boldsymbol{u}}^{w^{\mathrm{T}}}
\end{array}\right) \mid \widehat{\boldsymbol{u}} \in \widehat{\boldsymbol{U}}\right\} .
$$

Observe that for all $(x, y) \in R^{2}$, for all $\boldsymbol{u} \in \boldsymbol{U}$, and all $\widehat{\boldsymbol{u}} \in \widehat{\boldsymbol{U}}$ we have

$$
\left(\boldsymbol{u}^{x}, \boldsymbol{u}^{y}\right)\left(\begin{array}{c}
\widehat{\boldsymbol{u}}^{v^{\mathrm{T}}} \\
\widehat{\boldsymbol{u}}^{w^{\mathrm{T}}}
\end{array}\right)=\left\langle\boldsymbol{u}^{x}, \widehat{\boldsymbol{u}}^{v^{\mathrm{T}}}\right\rangle+\left\langle\boldsymbol{u}^{y}, \widehat{\boldsymbol{u}}^{w^{\mathrm{T}}}\right\rangle=\left\langle\boldsymbol{u}^{x v+y w}, \widehat{\boldsymbol{u}}\right\rangle .
$$

Let us write $\widehat{\boldsymbol{Y}}$ for the set on the right hand side of (24). Equation (25) for $(x, y):=(a, b)$ says that $\boldsymbol{U}^{(a, b)} \subseteq \widehat{\boldsymbol{Y}}^{\perp}$, since $a v+b w=0$. Likewise, (25) for $(x, y):=(c, d)$ gives the reverse inclusion as follows: We have $c v+d w=1$. Thus for each non-trivial linear form in $\widehat{\boldsymbol{Y}}$ there is a non-zero vector of $\boldsymbol{U}^{(c, d)}$ which is not in its kernel. This means that $\widehat{\boldsymbol{Y}}^{\perp}$ is contained in some complement of $\boldsymbol{U}^{(c, d)}$ and, by the above, this complement has to be $\boldsymbol{U}^{(a, b)}$. Altogether, we get $\widehat{\boldsymbol{Y}}^{\perp}=\boldsymbol{U}^{(a, b)}$ which is equivalent to (24). 
Choose any semilinear bijection $h: \widehat{U} \rightarrow \boldsymbol{U}^{\prime}$ with the same accompanying anti-isomorphism $K \rightarrow K^{\prime}$ as $f$. Then the mapping $f$ can be written as

$$
\left(\begin{array}{c}
\widehat{\boldsymbol{u}}_{1} \\
\widehat{\boldsymbol{u}}_{2}
\end{array}\right) \stackrel{f}{\longmapsto}\left(-\widehat{\boldsymbol{u}}_{2}^{h}, \widehat{\boldsymbol{u}}_{1}^{h}\right)\left(\begin{array}{ll}
g_{11} & g_{12} \\
g_{21} & g_{22}
\end{array}\right),
$$

with a matrix $\gamma:=\left(g_{i j}\right) \in \mathrm{GL}_{2}\left(R^{\prime}\right)$. Clearly, $\alpha: R \rightarrow R^{\prime}: x \mapsto h^{-1} x^{\mathrm{T}} h$ is an anti-isomorphism of rings. Now it is straightforward to show that $R(a, b)^{\varphi}=$ $R^{\prime}\left(-w^{\alpha}, v^{\alpha}\right)^{\widetilde{\gamma}}=R(a, b)^{\widetilde{\alpha} \widetilde{\gamma}}$.

We refer to [6, Thm. 5.1] for a similar result for the particular case that $\operatorname{dim} \boldsymbol{U}=$ $\operatorname{dim} \boldsymbol{U}^{\prime}=2$, where $\alpha$ is only assumed to be a bijective $\triangle$-morphism. (The description based on formula (9) in that theorem is erroneous; a correct version can be derived from (26) above.)

5.5 Corollary. Let $R$ be the ring of $n \times n$ matrices over a field $K$, and let $R^{\prime}$ be the ring of $n^{\prime} \times n^{\prime}$ matrices over a field $K^{\prime}$. Moreover, let $n>1$, and let $\omega: R \rightarrow R^{\prime}$ be a Jordan isomorphism. Then the following hold:

(a) $n=n^{\prime}$ and $\omega$ is either an isomorphism or an anti-isomorphism.

(b) If $\omega$ is an isomorphism, then there are an isomorphism $\beta: K \rightarrow K^{\prime}$ and a matrix $G \in R^{\prime *}$ such that $X^{\omega}=G^{-1} X^{\beta} G$ holds for all $X \in R$.

(c) If $\omega$ is an anti-isomorphism, then there are an anti-isomorphism $\beta: K \rightarrow K^{\prime}$ and a matrix $G \in R^{\prime *}$ such that $X^{\omega}=G^{-1}\left(X^{\beta}\right)^{\mathrm{T}} G$ holds for all $X \in R$.

(In cases (b) and (c) the mapping $\beta$ acts on the entries of the matrix $X$.)

Proof. Choose any matrix $X \in R$. By (5), the Jordan isomorphism $\omega$ induces a $\triangle$-isomorphism $\widetilde{\omega}: \mathbb{P}(R) \rightarrow \mathbb{P}\left(R^{\prime}\right)$. Putting $a=1 \in R$ and $b=X+1 \in R$ in (5) gives

$$
R(X, 1)^{\widetilde{\omega}}=R^{\prime}\left(X^{\omega}, 1\right) .
$$

We repeat the proof of Theorem 5.4 for $\varphi=\widetilde{\omega}, U=K^{n}, \boldsymbol{U} \times \boldsymbol{U}=K^{2 n}$ etc. This gives $n=n^{\prime}>1$ and there are accordingly two cases:

In case (i) let $\beta: K \rightarrow K^{\prime}$ be the accompanying automorphism of $f$. We may choose $h$ in such a way that its matrix is $1 \in R^{\prime}$. Hence $X^{\alpha}=X^{\beta}$, where $\beta$ acts on the entries of $X$. Since $\widetilde{\alpha}$ and $\widetilde{\omega}$ map $R(1,0), R(0,1)$, and $R(1,1)$ to $R^{\prime}(1,0)$, $R^{\prime}(0,1)$, and $R^{\prime}(1,1)$, respectively, we conclude that $\gamma=\operatorname{diag}(G, G)$ for a matrix $G \in R^{\prime *}$. This gives

$$
R(X, 1)^{\widetilde{\omega}}=R(X, 1)^{\tilde{\alpha} \widetilde{\gamma}}=R^{\prime}\left(X^{\beta} G, G\right)=R^{\prime}\left(G^{-1} X^{\beta} G, 1\right) .
$$

From (27) and (28) follows $X^{\omega}=G^{-1} X^{\beta} G$. So $\omega$ is an isomorphism of rings.

In case (ii) it can be shown similarly that $\omega$ is an anti-isomorphism with the required properties: In order to calculate $R(X, 1)^{\widetilde{\alpha}}$ according to 2.4 (c) one may use the matrix $M:=\left(\begin{array}{cc}X & 1 \\ 1 & 0\end{array}\right)$ so that $M^{-1}=\left(\begin{array}{cc}0 & 1 \\ 1 & -X\end{array}\right)$. 
The statement of Corollary 5.5 is well known: See [26, Thm. 3.24], where Jordan isomorphisms are called semi-isomorphisms instead. Of course, the assertion in (a) is also valid when $n=1$, provided that the word "either" is deleted from the text: In fact, then $n^{\prime}=1$, too, and we have a Jordan isomorphism of fields, which is an isomorphism or anti-isomorphism by Hua's theorem. See [26, Thm. 2.25].

\section{Direct products.}

6.1. Now we consider direct products of rings and the associated projective lines. Let $R=\prod_{i \in I} R_{i}$ be the direct product of a family $\left(R_{i}\right)_{i \in I}$ of rings, where it is tacitly assumed throughout this section that $I$ is non-empty. Then $\mathrm{GL}_{2}(R) \cong \prod_{i \in I} \mathrm{GL}_{2}\left(R_{i}\right)$, whence the points of $\mathbb{P}(R)$ are exactly the submodules $R\left(\left(a_{i}\right)_{i \in I},\left(b_{i}\right)_{i \in I}\right) \leq R^{2}$ with $R_{i}\left(a_{i}, b_{i}\right) \in \mathbb{P}\left(R_{i}\right)$ for all $i \in I$. So we can identify each $p \in \mathbb{P}(R)$ with a family $\left(p_{i}\right)_{i \in I}$ in the direct product (cartesian product) $\prod_{i \in I} \mathbb{P}\left(R_{i}\right)$ of the projective lines $\mathbb{P}\left(R_{i}\right)$, and vice versa.

As a general rule, we adopt the following notation: Given an $a \in R$ and an index $j \in I$ we write $a_{j}$ for the component of $a$ in $R_{j}$, i.e., we assume that $a=\left(a_{i}\right)_{i \in I}$. The same kind of notation is used whenever applicable, e.g. for points $p \in \mathbb{P}(R)$. Observe that, as before, we use the same symbols $(\Delta, \|, \sim)$ for the corresponding relations on all our projective lines.

6.2 Proposition. Let $R=\prod_{i \in I} R_{i}$. Identify $\mathbb{P}(R)$ with $\prod_{i \in I} \mathbb{P}\left(R_{i}\right)$ as above. Then the following statements hold for all $p, q$, and $r \in \mathbb{P}(R)$ :

(a) $p \triangle q \Leftrightarrow \forall i \in I: p_{i} \triangle q_{i}$.

(b) $p\left\|q \Leftrightarrow \forall i \in I: p_{i}\right\| q_{i}$.

(c) $p \sim q$ via $r \Leftrightarrow \exists j \in I:\left(p_{j} \sim q_{j}\right.$ via $r_{j}$ and $\left.\forall i \in I \backslash\{j\}: p_{i}\left\|q_{i}\right\| r_{i}\right)$.

Proof. (a): This is clear from $\mathrm{GL}_{2}(R) \cong \prod_{i \in I} \mathrm{GL}_{2}\left(R_{i}\right)$.

(b): This is immediate from (a) and the definition of parallel points.

(c): Let $p \sim q$ via $r$. Since $r \not k p$, by (b) there is at least one $j \in I$ such that $r_{j} \nVdash p_{j}$. This means that there is a point $y_{j} \in \mathbb{P}\left(R_{j}\right)$ with $y_{j} \triangle r_{j}$ and $y_{j} \not p_{j}$.

We show first that $r_{i} \| q_{i}$ if $i \neq j$ :

For each $i \neq j$ consider an arbitrary point $y_{i} \in \mathbb{P}\left(R_{i}\right)$ such that $y_{i} \triangle r_{i}$; at least one such $y_{i}$ exists. Then $y:=\left(y_{i}\right)_{i \in I} \Delta r$, whence $y \Delta p$ or $y \Delta q$ must hold by assumption. But $y \triangle p$ is impossible since $y_{j} \not p_{j}$. So $y \triangle q$, and in particular $y_{i} \triangle q_{i}$ for each $i \neq j$. Altogether $r_{i} \| q_{i}$ for $i \neq j$, as desired.

Since $r \nVdash q$, we conclude from (b) and the above that $r_{j} \nVdash q_{j}$ must hold. As before, we can now infer that $r_{i} \| p_{i}$ for $i \neq j$.

It remains to show, for all $x_{j} \in \mathbb{P}\left(R_{j}\right)$, that $x_{j} \Delta r_{j}$ implies $x_{j} \Delta p_{j}$ or $x_{j} \Delta q_{j}$. For each $i \neq j$ choose an $x_{i} \in \mathbb{P}\left(R_{i}\right)$ with $x_{i} \triangle r_{i}$. Then $x:=\left(x_{i}\right)_{i \in I} \triangle r$, and (a) gives the assertion. 
Conversely, let $p_{j} \sim q_{j}$ via $r_{j}$ for a $j \in I$ and $p_{i}\left\|q_{i}\right\| r_{i}$ for all $i \neq j$. By (b), we have $r \nVdash p, q$. Let $x \Delta r$. Then (a) and the assumption yield $x_{j} \Delta p_{j}$ or $x_{j} \Delta q_{j}$. On the other hand, for $i \neq j$, we get from (a) that $x_{i} \Delta p_{i}$, since $r_{i} \| p_{i}$, and likewise $x_{i} \triangle q_{i}$, since $r_{i} \| q_{i}$. So $x \triangle p$ or $x \triangle q$.

Note that statement (b) reflects the algebraic fact $\operatorname{rad}\left(\prod_{i \in I} R_{i}\right)=\prod_{i \in I} \operatorname{rad} R_{i}$ about Jacobson radicals; cf. 3.1 .

6.3. Let now $\left(\mathfrak{M}_{i}\right)_{i \in I}$ be a family of partial linear spaces $\mathfrak{M}_{i}=\left(\mathcal{P}_{i}, \mathcal{L}_{i}\right)$. The direct product (or Segre product) of the $\mathfrak{M}_{i}$ is the partial linear space $\mathfrak{M}:=\prod_{i \in I} \mathfrak{M}_{i}:=$ $(\mathcal{P}, \mathcal{L})$ with point set $\mathcal{P}:=\prod_{i \in I} \mathcal{P}_{i}$ and line set $\mathcal{L}:=\bigcup_{i \in I} \mathcal{L}_{(i)}$, where for each $j \in I$ we define

$$
\mathcal{L}_{(j)}:=\left\{\left\{x \in \mathcal{P} \mid x_{j} \in L_{j} \wedge \forall i \in I \backslash\{j\}: x_{i}=p_{i}\right\} \mid L_{j} \in \mathcal{L}_{j}, p \in \mathcal{P}\right\} .
$$

See [23]. Note that in this definition of a line, the point $p_{j} \in \mathbb{P}\left(R_{j}\right)$ is irrelevant.

6.4. Let $R=\prod_{i \in I} R_{i}$, where each $R_{i}$ is an endomorphism ring $\operatorname{End}_{K_{i}}\left(\boldsymbol{U}_{i}\right)$ of a left vector space over a field $K_{i}, 1 \leq \operatorname{dim} \boldsymbol{U}_{i}<\infty$. Let $\mathfrak{M}_{i}=\left(\mathcal{G}_{i}, \mathcal{L}_{i}\right)$ be the Grassmann space associated to $\mathbb{P}\left(R_{i}\right)$, and let $\Psi_{i}: \mathbb{P}\left(R_{i}\right) \rightarrow \mathcal{G}_{i}$ be given as in (15). We call

$$
\mathfrak{M}:=(\mathcal{G}, \mathcal{L}):=\prod_{i \in I} \mathfrak{M}_{i}
$$

the product space associated to $\mathbb{P}(R)$. Then, generalizing (15), the mapping

$$
\Psi: \mathbb{P}(R) \rightarrow \mathcal{G}: p \mapsto\left(p_{i}^{\Psi_{i}}\right)_{i \in I}
$$

is a bijection. According to our notational setting we have the trivial identity $\left(p^{\Psi}\right)_{i}=p_{i}^{\Psi_{i}}$ for all $i \in I$. We are now in a position to generalize Proposition 3.8 as follows:

6.5 Proposition. Let $R=\prod_{i \in I} R_{i}$, where $R_{i}=\operatorname{End}_{K_{i}}\left(\boldsymbol{U}_{i}\right)$ as above. Let $\mathfrak{M}$ be the product space associated to $\mathbb{P}(R)$. Then the following statements hold for all $p$, $q$, and $r \in \mathbb{P}(R)$, where $\Psi$ is given as in (31):

(a) $p \sim q \Leftrightarrow p^{\Psi}$ and $q^{\Psi}$ are distinct collinear points of $\mathfrak{M}$.

(b) $p \sim q$ via $r \Leftrightarrow p^{\Psi}, q^{\Psi}$, and $r^{\Psi}$ are distinct collinear points of $\mathfrak{M}$.

Proof. It suffices to prove (b), since every line of $\mathfrak{M}$ contains at least three points. By Proposition 6.2 (c), we have that $p \sim q$ via $r$ is equivalent to

$$
\exists j \in I:\left(p_{j} \sim q_{j} \text { via } r_{j} \text { and } \forall i \in I \backslash\{j\}: p_{i}=q_{i}=r_{i}\right) .
$$

(Recall that here $\|$ is equality.) By Proposition 3.8 (b), this is equivalent to

$$
\exists j \in I:\left\{\begin{array}{l}
\left(p^{\Psi}\right)_{j},\left(q^{\Psi}\right)_{j},\left(r^{\Psi}\right)_{j} \text { are distinct collinear points of } \mathfrak{M}_{j} \\
\text { and } \forall i \in I \backslash\{j\}:\left(p^{\Psi}\right)_{i}=\left(q^{\Psi}\right)_{i}=\left(r^{\Psi}\right)_{i} .
\end{array}\right.
$$


Finally, by (29), this is means that $p^{\Psi}, q^{\Psi}$, and $r^{\Psi}$ are distinct collinear points of $\mathfrak{M}$.

In the following results we confine ourselves to finite (non-empty) products. In case of an infinite product, the space $\mathfrak{M}$ is not connected, and our proofs for Proposition 6.6, Theorem 6.7, and Theorem 7.2 are not applicable. So the rings in the following proposition and the subsequent theorem are exactly the semisimple rings.

6.6 Proposition. Let $R=\prod_{i=1}^{m} R_{i}$ and $R^{\prime}=\prod_{j=1}^{m^{\prime}} R_{j}^{\prime}$ with $R_{i}=\operatorname{End}_{K_{i}}\left(\boldsymbol{U}_{i}\right)$, $R_{j}^{\prime}=\operatorname{End}_{K_{j}^{\prime}}\left(\boldsymbol{U}_{j}^{\prime}\right)$, where $1 \leq \operatorname{dim} \boldsymbol{U}_{i}, \operatorname{dim} \boldsymbol{U}_{j}^{\prime}<\infty$. Let $\mathfrak{M}$ and $\mathfrak{M}^{\prime}$ be the associated product spaces and suppose that $\Psi$ and $\Psi^{\prime}$ are given according to (31). For a mapping $\varphi: \mathbb{P}(R) \rightarrow \mathbb{P}\left(R^{\prime}\right)$, the following statements are equivalent:

(a) $\varphi$ is a $\triangle$-isomorphism.

(b) $\varphi$ is a $\sim$-isomorphism.

(c) $\Psi^{-1} \varphi \Psi^{\prime}: \mathcal{G} \rightarrow \mathcal{G}^{\prime}$ is a collineation $\mathfrak{M} \rightarrow \mathfrak{M}^{\prime}$.

Proof. (a) $\Rightarrow(\mathrm{c})$ : By definition, for all $p, q$ and $r$ in $\mathbb{P}(R)$ we have $p \sim q$ via $r$ if, and only if, $p^{\varphi} \sim q^{\varphi}$ via $r^{\varphi}$. Recall that every line of $\mathfrak{M}$ has at least three distinct points. The same applies to $\mathbb{P}\left(R^{\prime}\right)$ and $\mathfrak{M}^{\prime}$. Hence the characterization of collinear points in Proposition 6.5 (b) gives the required result.

(c) $\Rightarrow(\mathrm{b})$ : This is immediate from Proposition 6.5 (a).

(b) $\Rightarrow$ (a): For each $i \in\{1,2, \ldots, m\}$ let us consider $\mathbb{P}\left(R_{i}\right)$ as the set of vertices of the so-called adjacency graph, where two vertices are joined by an edge if, and only if, they are adjacent. The distance function in this graph will be written as dist. By Proposition 3.8 (a), the bijection $\Psi_{i}$ is an isomorphism of the adjacency graph on $\mathbb{P}\left(R_{i}\right)$ onto the Grassmann graph on $\mathcal{G}_{i}$. A simple induction shows that

$$
\forall p_{i}, q_{i} \in \mathbb{P}\left(R_{i}\right): \operatorname{dist}\left(p_{i}, q_{i}\right)=\operatorname{dim} \boldsymbol{U}_{i}-\operatorname{dim}\left(p_{i}^{\Psi_{i}} \cap q_{i}^{\Psi_{i}}\right) \leq \operatorname{dim} \boldsymbol{U}_{i} .
$$

The maximal distance $\operatorname{dim} \boldsymbol{U}_{i}$ is assumed precisely when $p_{i} \Delta q_{i}$.

Similarly, $\mathbb{P}(R)$ and its adjacency relation give rise to an adjacency graph, and (34), together with Proposition 6.2 (c), yields the following formula:

$$
\forall p, q \in \mathbb{P}(R): \operatorname{dist}(p, q)=\sum_{i=1}^{m} \operatorname{dist}\left(p_{i}, q_{i}\right) \leq \sum_{i=1}^{m} \operatorname{dim} \boldsymbol{U}_{i}
$$

By Proposition 6.2 (a), here the points $p$ and $q$ are distant, if, and only if, their distance attains the bound given in (35).

Let now $\varphi$ be a $\sim$-isomorphism. Then two points of $\mathbb{P}(R)$ with maximal distance (in the adjacency graph) go over to points of $\mathbb{P}\left(R^{\prime}\right)$ with maximal distance and vice versa. By the above, this means that $\varphi$ is a $\triangle$-isomorphism. 
This brings us to our main result:

6.7 Theorem. Let $R=\prod_{i=1}^{m} R_{i}$ and $R^{\prime}=\prod_{j=1}^{m^{\prime}} R_{j}^{\prime}$ with $R_{i}=\operatorname{End}_{K_{i}}\left(\boldsymbol{U}_{i}\right), R_{j}^{\prime}=$ $\operatorname{End}_{K_{j}^{\prime}}\left(\boldsymbol{U}_{j}^{\prime}\right)$, where $1 \leq \operatorname{dim} \boldsymbol{U}_{i}, \operatorname{dim} \boldsymbol{U}_{j}^{\prime}<\infty$. For a mapping $\varphi: \mathbb{P}(R) \rightarrow \mathbb{P}\left(R^{\prime}\right)$, the following statements are equivalent:

(a) $\varphi$ is a $\triangle$-isomorphism.

(b) $m=m^{\prime}$, and there is a permutation $\sigma$ of $\{1,2, \ldots, m\}$ such that for each $p \in \mathbb{P}(R)$ and each $k \in\{1,2, \ldots, m\}$ we have $\left(p^{\varphi}\right)_{k^{\sigma}}=\left(p_{k}\right)^{\varphi_{k}}$ for $\Delta$-isomorphisms $\varphi_{k}: \mathbb{P}\left(R_{k}\right) \rightarrow \mathbb{P}\left(R_{k^{\sigma}}^{\prime}\right)$.

In this case, if $\operatorname{dim} \boldsymbol{U}_{i}>1$ for all $i \in\{1,2, \ldots, m\}$, then $\varphi=\widetilde{\alpha} \widetilde{\gamma}$, where $\gamma \in$ $\mathrm{GL}_{2}\left(R^{\prime}\right)$ and $\alpha: R \rightarrow R^{\prime}$ is a Jordan isomorphism such that for each $x \in R$ and each $k \in\{1,2, \ldots, m\}$ we have $\left(x^{\alpha}\right)_{k^{\sigma}}=\left(x_{k}\right)^{\alpha_{k}}$, with $\alpha_{k}: R_{k} \rightarrow R_{k^{\sigma}}^{\prime}$ an isomorphism or an anti-isomorphism.

Proof. (a) $\Rightarrow$ (b): By Proposition 6.6, the mapping $\Psi^{-1} \varphi \Psi^{\prime}: \mathcal{G} \rightarrow \mathcal{G}^{\prime}$ is a collineation $\mathfrak{M} \rightarrow \mathfrak{M}^{\prime}$. Since each $\mathfrak{M}_{i}$ is strongly connected and contains at least one line, the assertion follows from Theorem 7.2 in the Appendix and Proposition 5.2.

While (b) $\Rightarrow$ (a) is obvious, the other assertions follow from Theorem 5.4.

From this and Proposition 4.4 we obtain the following:

6.8 Corollary. Let $R$ and $R^{\prime}$ be semilocal rings. Then a bijection $\varphi: \mathbb{P}(R) \rightarrow$ $\mathbb{P}\left(R^{\prime}\right)$ is a $\triangle$-isomorphism if, and only if, it is a $\|$-isomorphism such that the induced bijection $\bar{\varphi}: \mathbb{P}(\bar{R}) \rightarrow \mathbb{P}\left(\overline{R^{\prime}}\right)$ (see (19)) is a mapping as in Theorem 6.7.

From Theorem 6.7, Corollary 5.5 (a) and Hua's theorem [26, Thm. 2.25], or from Theorem 6.7 and [26, Thm. 3.24], we infer the following algebraic description of Jordan isomorphisms between semisimple rings.

6.9 Corollary. Let $R=\prod_{i=1}^{m} R_{i}$ and $R^{\prime}=\prod_{j=1}^{m^{\prime}} R_{j}^{\prime}$ with $R_{i}, R_{j}^{\prime}$ matrix rings over fields. Let $\omega: R \rightarrow R^{\prime}$ be a Jordan isomorphism. Then $m=m^{\prime}$, and there is a permutation $\sigma$ of $\{1,2, \ldots, m\}$ such that for each $x \in R$ and each $k \in\{1,2, \ldots, m\}$ we have $\left(x^{\omega}\right)_{k^{\sigma}}=\left(x_{k}\right)^{\omega_{k}}$, where $\omega_{k}: R_{k} \rightarrow R_{k^{\sigma}}^{\prime}$ is an isomorphism or an anti-isomorphism.

Note that this can also be shown in a purely algebraic way: Just replace "Theorem 2" with "[26, Thm. 3.24]" in the proof of [19, Thm. 3], where a similar result is shown for direct products of finitely many simple algebras of finite dimension. 


\section{Appendix.}

7.1. The following is a generalization of [23, Prop. 1.10], as we consider not only automorphisms but also isomorphisms of product spaces. For the reader's convenience we stick close to the notation used in [23]:

7.2 Theorem. Let $\mathfrak{M}_{i}=\left(X_{i}, \mathcal{L}_{i}\right), i \in\{1,2, \ldots, m\}$, and $\mathfrak{M}_{j}^{\prime}=\left(X_{j}^{\prime}, \mathcal{L}_{j}^{\prime}\right), j \in$ $\left\{1,2, \ldots, m^{\prime}\right\}$ be strongly connected partial linear spaces with at least one line, where $m, m^{\prime} \geq 1$. Suppose, furthermore, that

$$
f: \prod_{i=1}^{m} X_{i} \rightarrow \prod_{j=1}^{m^{\prime}} X_{j}^{\prime}
$$

is a collineation of $\mathfrak{M}:=\prod_{i=1}^{m} \mathfrak{M}_{i}$ onto $\mathfrak{M}^{\prime}:=\prod_{j=1}^{m^{\prime}} \mathfrak{M}_{j}^{\prime}$. Then the following assertions hold:

(a) $m=m^{\prime}$.

(b) There exists a permutation $\sigma$ of the set $\{1,2, \ldots, m\}$, and for each $k \in$ $\{1,2, \ldots, m\}$ there is a collineation $f_{k}: X_{k} \rightarrow X_{\sigma(k)}^{\prime}$ such that

$$
\left(f\left(c_{1}, c_{2}, \ldots, c_{m}\right)\right)_{\sigma(k)}=f_{k}\left(c_{k}\right)
$$

for all points $\left(c_{1}, c_{2}, \ldots, c_{m}\right) \in \prod_{i=1}^{m} X_{i}$.

Proof. (a): Let $S$ and $T$ be strong subspaces of $\mathfrak{M}$. Following [23, p. 131], we let $S \approx T$ if, and only if, there exists a finite sequence $Y_{0}, Y_{1}, \ldots, Y_{b}$ of strong subspaces such that $S=Y_{0}, T=Y_{b}$, and $\#\left(Y_{i} \cap Y_{i-1}\right) \geq 2$ for all $i \in\{1,2, \ldots, b\}$. This is an equivalence relation on the set of all strong subspaces.

Let us choose a point $p$ of $\mathfrak{M}$. We restrict $\approx$ to the (non-empty) set of strong subspaces which contain $p$ and at least one more point. By [23, Lemma 1.5], there are precisely $m$ equivalence classes of this restricted relation. Similarly, we have a relation $\approx^{\prime}$ on the set of all strong subspaces of $\mathfrak{M}^{\prime}$. There are precisely $m^{\prime}$ equivalence classes when $\approx^{\prime}$ is restricted to the set of strong subspaces of $\mathfrak{M}^{\prime}$ which contain a fixed point $p^{\prime}$ and at least one more point. As $f$ and $f^{-1}$ preserve strong subspaces with more than one point, and because of $S \approx T \Leftrightarrow f(S) \approx^{\prime} f(T)$, we finally get $m=m^{\prime}$.

(b): By virtue of (a), it is easy to see that Proposition 1.6, Corollary 1.9, and Proposition 1.10 in [23] hold, mutatis mutandis, even under our assumptions. As a matter of fact, the proofs given in [23] and the ones which are needed now are, up to notational changes, the same. Therefore the assertion follows.

Acknowledgement. The authors are grateful to Peter Šemrl (Ljubljana) and Matej Brešar (Maribor) for their kind assistance when searching for literature on Jordan homomorphisms. 


\section{References.}

[1] C. BARTOLOnE, Jordan homomorphisms, chain geometries and the fundamental theorem, Abh. Math. Sem. Univ. Hamburg 59 (1989), 93-99.

[2] C. Bartolone And F. BARTOlOzZI, Topics in geometric algebra over rings, In Rings and Geometry, R. Kaya, P. Plaumann, and K. Strambach, Eds., Reidel, Dordrecht, 1985, pp. 353-389.

[3] W. Benz, Geometrische Transformationen, Bibl. Institut, Mannheim, 1992.

[4] A. Blunck, Regular spreads and chain geometries, Bull. Belg. Math. Soc. Simon Stevin 6 (1999), 589-603.

[5] A. Blunck and H. Havlicek, Projective representations I. Projective lines over rings, Abh. Math. Sem. Univ. Hamburg 70 (2000), 287-299.

[6] A. Blunck and H. Havlicek, Projective representations II. Generalized chain geometries, Abh. Math. Sem. Univ. Hamburg 70 (2000), 301-313.

[7] A. Blunck And H. HavliceK, The connected components of the projective line over a ring, Adv. Geom. 1 (2001), 107-117.

[8] A. Blunck and H. Havlicek, The dual of a chain geometry, J. Geom. 72 (2001), 27-36.

[9] A. Blunck And H. HavliceK, Jordan homomorphisms and harmonic mappings, Monatsh. Math. 139 (2003), 111-127.

[10] A. Blunck And H. HavliceK, Radical parallelism on projective lines and non-linear models of affine spaces, Math. Pannonica 14 (2003), 113-127.

[11] A. Blunck And H. HavliceK, On bijections that preserve complementarity of subspaces, Discrete Math., in print.

[12] A. Brouner, A. Cohen, And A. Neumaier, Distance-Regular Graphs, Springer, Berlin Heidelberg New York, 1989.

[13] W.-L. CHow, On the geometry of algebraic homogeneous spaces, Ann. of Math. 50 (1949), $32-67$.

[14] T.-S. Fu And T. HuAng, A unified approach to a characterization of Grassmann graphs and bilinear form graphs, Eur. J. Comb. 15 (1994), 363-373.

[15] A. Herzer, On isomorphisms of chain geometries, Note Mat. 8 (1987), 251-270.

[16] A. Herzer, Chain geometries, In Handbook of Incidence Geometry, F. Buekenhout, Ed., Elsevier, Amsterdam, 1995, pp. 781-842.

[17] W.-L. Huang, Adjacency preserving transformations of Grassmann spaces, Abh. Math. Sem. Univ. Hamburg 68 (1998), 65-77.

[18] N. Jacobson, Structure and Representation of Jordan Algebras, Amer. Math. Soc., Providence, 1968.

[19] I. Kaplansky, Semi-automorphisms of rings, Duke Math. J. 14 (1947), 521-525.

[20] T.Y. LAM, A First Course in Noncommutative Rings, Springer, New York, 1991.

[21] S. Lang, Algebra, third ed., Addison-Wesley, Reading, Mass., 1995.

[22] A. LashKhi, Harmonic maps over rings, Georgian Math. J. 4 (1997), 41-64.

[23] A. Naumowicz and K. Prażmowski, On Segre's product of partial line spaces and spaces of pencils, J. Geom. 71 (2001), 128-143.

[24] M. Numata, A characterization of Grassmann and Johnson graphs, J. Comb. Theory, Ser. B 48 (1990), 178-190.

[25] F. VeldKamp, Geometry over rings, In Handbook of Incidence Geometry, F. Buekenhout, Ed., Elsevier, Amsterdam, 1995, pp. 1033-1084.

[26] Z.-X. Wan, Geometry of Matrices, World Scientific, Singapore, 1996. 
Andrea Blunck

Fachbereich Mathematik

Universität Hamburg

Bundesstraße 55

D-20146 Hamburg

Germany

email: andrea.blunck@math.uni-hamburg.de

Hans Havlicek

Institut für Diskrete Mathematik und Geometrie

Technische Universität

Wiedner Hauptstraße 8-10

A-1040 Wien

Austria

email: havlicek@geometrie.tuwien.ac.at 